\title{
Caracterización de residuos sólidos procedentes de dos procesos distintos de galvanizado en caliente por inmersión( ${ }^{(\cdot)}$
}

\author{
P. Delvasto*, J. A. Casal-Ramos*, O. González-Jordán*, N. C. Durán-Rodríguez*, J. R. Domínguez** \\ y P. Moncada***
}

\begin{abstract}
Resumen Se caracterizaron polvos y escorias de zinc de industrias venezolanas de galvanizado en caliente por inmersión empleando espectroscopía atómica, microscopía electrónica de barrido, difracción de R-X y espectroscopía infrarroja. Los polvos se obtienen del proceso de secado a alta presión de las piezas en una planta cuyo proceso se basa en una cuba de acero para contener el zinc fundido, planta de la cual procede también la muestra de escoria denominada "A". Por su parte, la escoria denominada "B" se genera en una industria que utiliza una cuba recubierta con refractario cerámico. Los polvos contienen $98 \%$ en peso de zinc, en forma metálica. Las escorias, por su parte, presentaron las siguientes fases: $\mathrm{Zn}$ y $\mathrm{ZnO}$,. Adicionalmente, la escoria "B" mostró la presencia $\mathrm{ZnCl}_{2}$ y $\mathrm{Zn}_{5}(\mathrm{OH})_{8} \mathrm{Cl}_{2} \times \mathrm{H}_{2} \mathrm{O}$. El contenido total de zinc (\% en peso) en la escoria "A" es $71 \%$, mientras que en la escoria "B" es $75 \%$.
\end{abstract}

Palabras clave Caracterización; Residuos; Galvanizado en caliente por inmersión; Escoria de zinc; Polvos de zinc.

\section{Characterization of solid wastes from two different hot-dip galvanizing processes}

\begin{abstract}
Zinc dust and zinc ash from hot-dip galvanizing industries located in Venezuela were characterized using atomic spectroscopy, scanning electron microscopy, X-Ray diffraction and infrared spectroscopy. Dust was formed during the high-pressure drying process of the galvanized pieces, in a plant that uses a steel kettle to hold the molten zinc. Ash identified as "A" came from the same plant as the dust, while ash identified as "B" came from a hot-dip galvanizing plant which use a ceramic lined galvanizing furnace. Dust contained $98 \mathrm{wt} \% \mathrm{Zn}$, in metallic form. Both ash samples contained: $\mathrm{Zn}$ and $\mathrm{ZnO}$, while $\mathrm{Zn}_{5}(\mathrm{OH})_{8} \mathrm{Cl}_{2} \times \mathrm{H}_{2} \mathrm{O}$ and $\mathrm{ZnCl}_{2}$ were only found in ash "B". Globally, ash "A" and ash "B" contain 71 and 75 wt \% Zn, respectively.
\end{abstract}

Keywords

Characterization; Wastes; Hot-dip galvanizing; Zinc ash; Zinc dust.

\section{INTRODUCCION}

Según cifras de la Asociación Latinoamericana de zinc, LATIZA (http://www.latiza.com), para el año 2009 se consumieron en el mundo más de 7,9 millones de TM de zinc, de las cuales, de acuerdo con datos de la International Zinc Association, IZA (http://www.zinc.org), aproximadamente un $50 \%$ se dirigió al mercado del galvanizado. Esta industria emplea el zinc como recubrimiento anticorrosivo para el acero, primordialmente, a través de un proceso de inmersión de las piezas de acero en zinc fundido, del cual se extraen las mismas con una capa de zinc típica que ronda las $120 \mu \mathrm{m}$ de espesor. De acuerdo con cifras de la Asociación Venezolana de Galvanizadores, AVGAL (http://www.avgal.net), en Venezuela, alrededor del $68 \%$ del zinc que se consume es de tipo primario, el mismo que fundamentalmente se destina a la industria del galvanizado en caliente. A falta de cifras más precisas, se infiere que cerca de 7500 TM de zinc se destinan anualmente al galvanizado en caliente en Venezuela.

Las plantas de galvanizado en caliente por inmersión producen una gran diversidad de material residual ${ }^{[1}$ y 2$]$. En particular, durante la etapa de formación del recubrimiento anticorrosivo, se generan tres clases

(.) Trabajo recibido el día 7 de julio de 2011 y aceptado en su forma definitiva el 23 de noviembre de 2011.

* Departamento de Ciencia de los Materiales, Universidad Simón Bolívar, Caracas, Venezuela, E-mail: delvasto@usb.ve

** Laboratorio de Espectroscopía Atómica, Universidad Simón Bolívar, Caracas, Venezuela.

*** Laboratorio de Química Instrumental, Universidad Simón Bolívar, Caracas, Venezuela. 
diferentes de residuos sólidos ricos en zinc: matas, escorias y polvos ${ }^{[1]}$. Las matas son compuestos intermetálicos, que se forman entre el zinc y el hierro, los cuales debido a su elevada densidad, se sedimentan en el fondo de la cuba que contiene el zinc líquido. Las escorias, por su parte se forman en la interfase entre la superficie del baño de zinc líquido y la atmósfera de la cuba, debido a las reacciones que ocurren entre el oxígeno, los elementos componentes del baño y los aditivos fundentes. Finalmente, los polvos se generan como consecuencia de los procesos de secado, escurrido y regulación de espesores de zinc de las piezas de acero recién extraídas del baño fundido. En dichos procesos se suele proyectar vapor de agua a altas presiones sobre las piezas galvanizadas, razón por la que se atomiza el zinc, el cual luego se recolecta, en forma de polvos, dentro de los sistemas de captación de gases de la cuba (ciclones y filtros de manga).

La cantidad generada de esta clase de residuos depende de cómo se lleve a cabo el proceso de galvanizado en cada planta. No obstante, esta clase de datos son difíciles de obtener, debido a las reservas que presentan algunos industriales para darlos a conocer, ya que tales cifras constituyen un reflejo de la eficiencia de su proceso productivo en particular. Estudios previos ${ }^{[1]}$ indican que valores típicos para una producción anual de 11.500 TM de productos de acero galvanizado implican un consumo de cerca de 750 TM de zinc anuales, de las cuales, sólo el $54 \%$ se refleja de manera efectiva, en forma de recubrimientos sobre los productos galvanizados. El restante $46 \%$ de zinc va a parar a los residuos generados por el proceso, distribuyéndose aproximadamente de la siguiente forma: $4 \%$ en las matas, $17 \%$ en las escorias y $19 \%$ en los polvos, el restante $6 \%$ se considera que corresponde a recubrimientos que no pasan los controles de calidad.

Éstos residuos sólidos del proceso de galvanizado poseen una fracción metálica aprovechable que generalmente, es reciclada por los proveedores del zinc primario, sin embargo, poco se sabe acerca del destino que se da a las fracciones no metálicas contenidas en estos materiales. Dichas fracciones, no directamente aprovechables, presentan contenidos muy elevados en metales pesados, los cuales pueden generar un impacto negativo sobre los ecosistema[ ${ }^{[3]}$, si no están dispuestos de manera segura. Por estos motivos, resulta fundamental caracterizar los residuos sólidos del proceso de galvanizado de una manera precisa. Dicha caracterización constituye además un paso inicial para establecer procesos factibles de valorización de esta clase de residuos.

Con base en la realidad anterior, en el presente trabajo se caracterizan química, morfológica y mineralógicamente dos tipos de residuos sólidos proce- dentes de industrias venezolanas de galvanizado en caliente por inmersión, conocidos como escorias y polvos de galvanizado. A tales fines, se emplean las técnicas de análisis químico por espectroscopía atómica (AS), microscopía electrónica de barrido (SEM), microanálisis químico por espectroscopía de energías dispersivas (EDS), difracción de R-X (XRD) y espectroscopía infrarroja (FTIR). El propósito es generar información básica que pueda facilitar procesos de toma de decisión referentes al aprovechamiento, valorización o disposición final apropiada de este tipo de residuos.

\section{PROCEDIMIENTO EXPERIMENTAL}

\subsection{Origen de los residuos a caracterizar}

\subsubsection{Polvos de galvanizado}

El polvo residual estudiado en este trabajo procede de una empresa galvanizadora ubicada en la región central de Venezuela. El proceso de galvanizado en esta empresa se caracteriza por emplear una cuba de acero, calentada por quemadores de gas natural, para el mantenimiento del zinc fundido. Este tipo de polvo se genera al momento de regular el espesor de la capa de zinc sobre el acero recién galvanizado. El procedimiento de regulación se lleva a cabo mediante la proyección de un chorro de aire y vapor de agua, a elevada presión, sobre la pieza de acero, lo que genera una corriente de vapor con gotas de zinc que son luego capturadas, ya solidificadas, por los ciclones y filtros de manga que constituyen el sistema de limpieza de gases de la planta. Los polvos recolectados son luego almacenados en contenedores para su disposición posterior.

\subsubsection{Escoria "A"}

Se denomina escoria "A" a un material residual procedente de la misma planta de galvanizado de la que se obtuvieron los polvos antes descritos en el apartado 2.1.1. Este residuo aparece en la superficie del baño de zinc fundido contenido en la cuba de acero, como consecuencia de las reacciones químicas que ocurren entre los componentes del baño, el oxígeno de la atmósfera, los fundentes remanentes empleados en los procesos previos de limpieza y los elementos componentes de la cuba y de las piezas de acero. Cabe señalar que en el proceso que da lugar a la escoria 
"A", la cuba de zinc fundido se mantiene alrededor de $450^{\circ} \mathrm{C}$.

\subsubsection{Escoria " $B$ "}

El residuo denominado escoria "B", procede de otra planta de galvanizado en caliente por inmersión, la cual emplea en su proceso una cuba recubierta con refractario cerámico de alta densidad, como contenedor del baño de zinc fundido. En este caso, el baño de zinc puede encontrarse a una temperatura variable, entre $470-650^{\circ} \mathrm{C}$, dependiendo del tipo de acero y pieza a recubrir. De igual manera, este residuo se genera por las reacciones químicas que se explican en el apartado 2.1.2.

\subsection{Caracterización de los residuos estudiados}

\subsubsection{Picnometría}

La medición de la densidad de cada uno de los residuos se realizó siguiendo la norma ASTM D2320-98. La temperatura registrada en el procedimiento fue de $25^{\circ} \mathrm{C}$.

\subsubsection{Espectroscopía atómica (AS)}

Se disolvieron $100 \mathrm{mg}$ de cada residuo en $10 \mathrm{~mL}$ de $\mathrm{HCl}$ al $37 \%$ en peso y se aforó a $500 \mathrm{~mL}$ con agua destilada. Seguidamente, esta solución madre se diluyó con agua destilada, antes de la determinación instrumental de los analitos mediante la técnica de AS, con un espectrofotómetro Perkin-Elmer 2380. Se destaca la implementación específica de espectroscopía de absorción atómica (AAS) para la medición de zinc, hierro, manganeso, cobre, cromo, plomo, magnesio y calcio. Por su parte, para la determinación del sodio, se empleó el mismo espectrofotómetro en modo de espectroscopía de emisión atómica (AES), para lo cual se añadió a la solución del residuo $\mathrm{CsCl}_{2}$ como supresor de ionización.

\subsubsection{Microscopía electrónica de barrido (SEM) con espectroscopía de energías dispersivas (EDS)}

Se recubrieron los residuos con oro o con platino mediante sputtering, antes de su inserción en un microscopio electrónico Hitachi S-2400, que operó a un voltaje de aceleración de $20 \mathrm{kV}$. Dicho equipo permitió la observación morfológica de los polvos y las escorias y el análisis microquímico elemental semi-cuantitativo por EDS. La determinación del tamaño medio de partícula de los polvos de galvanizado $\left(\mathrm{d}_{50}\right)$ se realizó mediante un software de análisis de imágenes.

\subsubsection{Difracción de rayos $\times(X R D)$}

La detección de las fases mineralógicas (cristalinas) presentes en los residuos estudiados en este trabajo, se llevó a cabo con un difractómetro Phillips XPERT Pro, empleando una longitud de onda incidente correspondiente a la radiación $\mathrm{k}$-alfa del cobalto $(1,7889700 \AA)$, un barrido desde $2 \theta=0^{\circ}$ hasta $2 \theta=140^{\circ}$, una velocidad de barrido igual a $0,012 \theta / \mathrm{s}$, un voltaje de $40 \mathrm{kV}$ y una corriente de $20 \mathrm{~mA}$. La identificación de las fases se realizó por comparación directa de los difractogramas obtenidos con las fichas estándar correspondientes, disponibles en la base de datos de la JCPDS-ICDD (Joint Committee on Powder Diffraction Standards - International Centre for Diffraction Data).

\subsubsection{Espectroscopía infrarroja por transformada de Fourier (FTIR)}

Para el análisis FTIR, las muestras fueron trituradas en un mortero de ágata y mezcladas con $\mathrm{KBr}$ en una relación $\mathrm{KBr}$ :muestra de 99:1. La mezcla se prensó manualmente hasta formar pastillas traslúcidas para cada residuo, las cuales fueron colocadas en un espectrómetro BRUKER Tensor 27 para la obtención de los espectros infrarrojos y su posterior corrección de la línea base. Las condiciones de operación del equipo fueron las siguientes: 16 barridos consecutivos y una resolución espectral de $2 \mathrm{~cm}^{-1}$. Al finalizar, las bandas de frecuencia fueron caracterizadas por comparación con la literatura.

\section{RESULTADOS Y DISCUSIONES}

\subsection{Polvos de galvanizado}

El polvo capturado por los sistemas de limpieza de gases de la cuba, presenta un aspecto granular y con un color predominantemente gris. La densidad, calculada mediante picnometría, se muestra en la 
tabla I. Como podrá verse más adelante, los polvos residuales están constituidos en su casi totalidad por zinc metálico, razón por la cual se puede atribuir que la diferencia entre la densidad de los polvos y la densidad tabulada del zinc metálico puro $\left(7,1 \mathrm{~g} / \mathrm{cm}^{3}\right)$, se debe a la eventual presencia, minoritaria, de óxido de zinc en los polvos.

El análisis químico elemental de los polvos de galvanizado, obtenido mediante AS, se presenta en la tabla II, observándose que los mismos se encuentran constituidos en su mayoría por zinc. Por otro lado, en la figura 1, se muestran las imágenes SEM del polvo de galvanizado, cuyas magnificaciones originales corresponden a $1000 \mathrm{X}$ y a $4000 \mathrm{X}$, en las imágenes (a) y (b), respectivamente. Las imágenes SEM revelan la morfología de los polvos estudiados,

Tabla I. Densidad de cada uno de los residuos de galvanizado estudiados, obtenida mediante picnometría a $25^{\circ} \mathrm{C}$

Table I. Density of each of the studied galvanizing residues, obtained by the pycnometer method at $25^{\circ} \mathrm{C}$

\section{Residuo Densidad $\pm 0,1\left(\mathrm{~g} / \mathrm{cm}^{3}\right)$}

\begin{tabular}{ll}
\hline Polvos de galvanizado & 6,7 \\
Escoria "A" & 4,7 \\
Escoria "B" & 5,6 \\
\hline
\end{tabular}

los cuales están constituidos por partículas esferoidales delimitadas por caras hexagonales, producto de la manifestación del hábito cristalino del zinc metálico. Adicionalmente, se encontró que el tamaño medio $\left(\mathrm{d}_{50}\right)$ de las partículas del polvo estaba alrededor de 16,5 $\mu \mathrm{m}$. El recuadro de la figura 1(b) encierra la zona en donde se evidencia deformación plástica de algunos granos, la cual es ocasionada durante las colisiones que sufren las partículas entre sí y con las paredes de los ductos y ciclones del sistema de captación de polvos de la cuba de galvanizado, cuando las mismas aún están calientes y en estado pastoso. Las circunferencias que aparecen en la figura 1 (a), indican las zonas en donde se realizó el microanálisis químico elemental semicuantitativo por EDS. Estos resultados se muestran normalizados en la tabla III y los espectros de los puntos 1 y 5 pueden observarse en las figuras 1 (c) y $1(\mathrm{~d})$, respectivamente. A pesar de no poseer un carácter cuantitativo en su totalidad, esta técnica complementa a los estudios químicos realizados por AS, corroborando una mayoritaria presencia de zinc en los polvos y, además, detectando aluminio como elemento adicional a los reportados en la tabla II. Si bien este metal no fue cuantificado mediante AS, se sabe que en el proceso empleado en la planta de la que se obtuvieron los polvos, a la cuba se añade entre 0,04 y $0,07 \%$ de aluminio, como agente modificador, lo cual explicaría la presencia de dicho elemento en los polvos residuales.

Las fases mineralógicas cristalinas presentes en los polvos fueron detectadas de manera cualitativa

Tabla II. Análisis químico elemental (\% en peso) por AS de todos los residuos de galvanizado estudiados en este trabajo

Table II. Elemental chemical analysis (wt \%) by AS of all the galvanizing residues studied in this work

\begin{tabular}{lccc}
\hline & $\begin{array}{c}\text { Polvo de } \\
\text { galvanizado }\end{array}$ & Escoria "A" & Escoria "B" \\
\hline $\mathrm{Zn}$ & $98 \pm 1$ & $71 \pm 4$ & $75 \pm 2$ \\
$\mathrm{Fe}$ & $0,09 \pm 0,01$ & $0,30 \pm 0,01$ & $8,3 \pm 0,4$ \\
$\mathrm{Mn}$ & $\mathrm{ND}^{*}$ & $0,021 \pm 0,02$ & $1,3 \pm 0,1$ \\
$\mathrm{Cu}$ & $\mathrm{ND}^{*}$ & $0,004 \pm 0,001$ & $0,00515 \pm 0,00001$ \\
$\mathrm{Cr}$ & $\mathrm{ND}^{*}$ & $\mathrm{ND}^{*}$ & $\mathrm{ND}$ \\
$\mathrm{Pb}$ & $\mathrm{ND}^{*}$ & $0,020 \pm 0,001$ & $0,000378 \pm 0,000003$ \\
$\mathrm{Mg}$ & $0,007 \pm 0,001$ & $0,0045 \pm 0,0001$ & $0.0050 \pm 0.0003$ \\
$\mathrm{Ca}$ & $0,010 \pm 0,001$ & $0,027 \pm 0,003$ & $0,029 \pm 0,003$ \\
$\mathrm{Na}$ & $0,014 \pm 0,004$ & $0,077 \pm 0,003$ & $0,037 \pm 0,003$ \\
$\mathrm{~K}$ & $0,006 \pm 0,004$ & $0,0189 \pm 0,0001$ & $0,0120 \pm 0,0003$ \\
\hline
\end{tabular}

${ }^{*}$ ND: No detectado.

${ }^{*}$ ND: Not detected. 

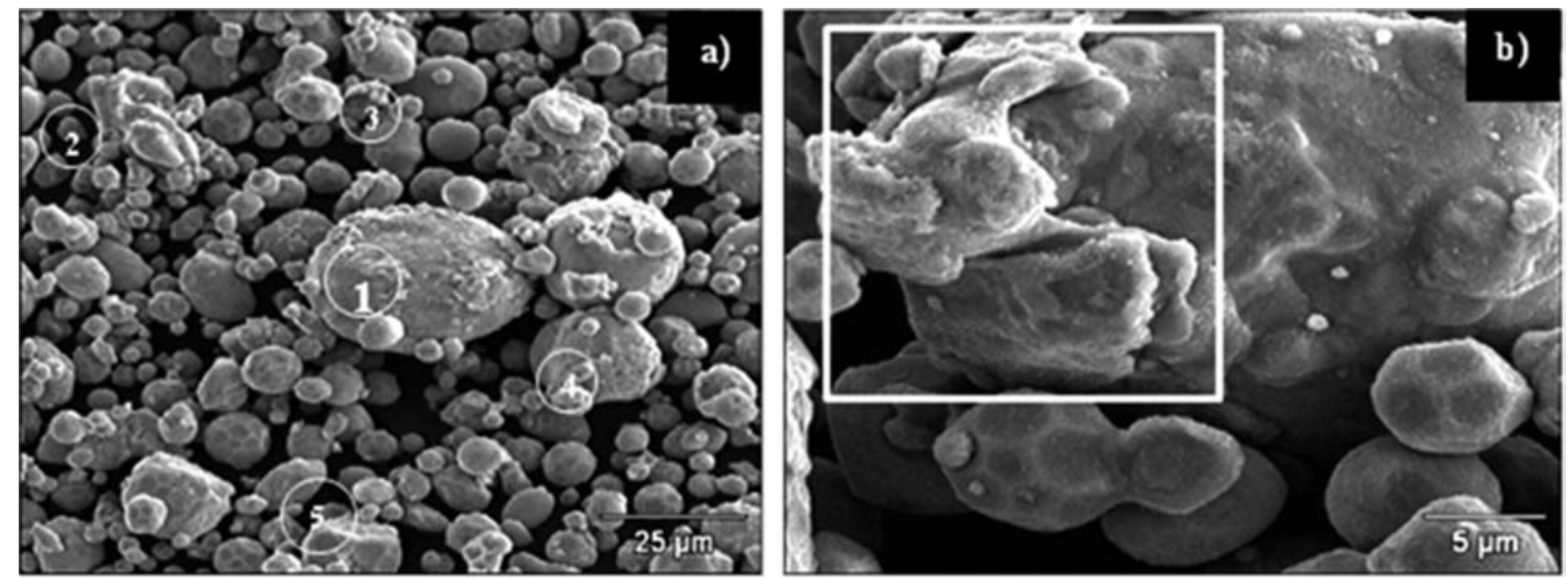

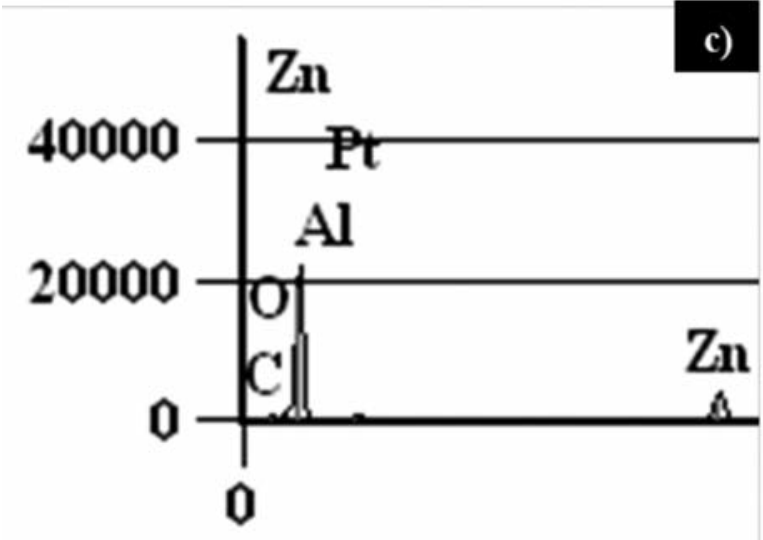

keV

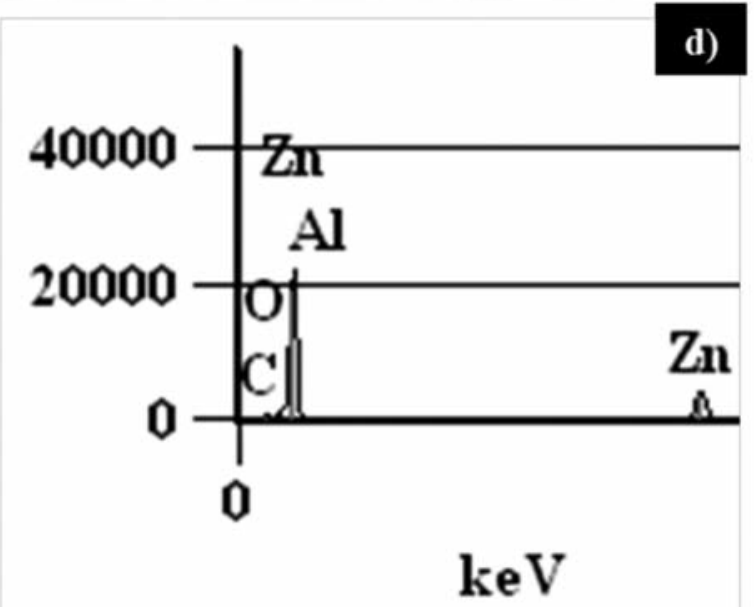

Figura 1. Imágenes SEM y espectros EDS del polvo de galvanizado.

Figure 1. SEM images and EDS spectra of the galvanizing dust.

empleando la técnica de XRD. El difractograma típico de este residuo se muestra en la figura 2 y en el mismo se observa que la fase mayoritaria presente es el zinc metálico (Zn). Estos resultados concuerdan con el hecho de que los polvos residuales provengan del material removido durante el proceso de secado, escurrido y de regulación de espesores de las piezas recién galvanizadas, mediante el uso de un chorro a presión de vapor de agua y aire. En este proceso se arrastra parte de la capa más exterior del recubrimiento de galvanizado, la cual está compuesta, en su práctica totalidad, por zinc metálico ${ }^{[4]}$, el cual, al encontrarse inicialmente en estado líquido, conforma gotas, las cuales dan lugar a las formas esféricas que exhibe el

Tabla III. Microanálisis químico elemental semicuantitativo por EDS (\% en peso) realizado sobre las zonas indicadas en la figura 1(a) para los polvos de galvanizado

Table III. Semiquantitative elemental chemical microanalysis by EDS (wt \%) performed on the areas shown in figure 1(a) for galvanizing dust

\begin{tabular}{lccccc}
\hline & Zona 1 & Zona 2 & Zona 3 & Zona 4 & Zona 5 \\
\hline Zn & 99,75 & 99,69 & 99,93 & 100,00 & 99,90 \\
$\mathrm{Al}$ & 0,25 & 0,20 & 0,07 & - & 0,10 \\
$\mathrm{Ca}$ & - & 0,11 & - & - & - \\
\hline
\end{tabular}




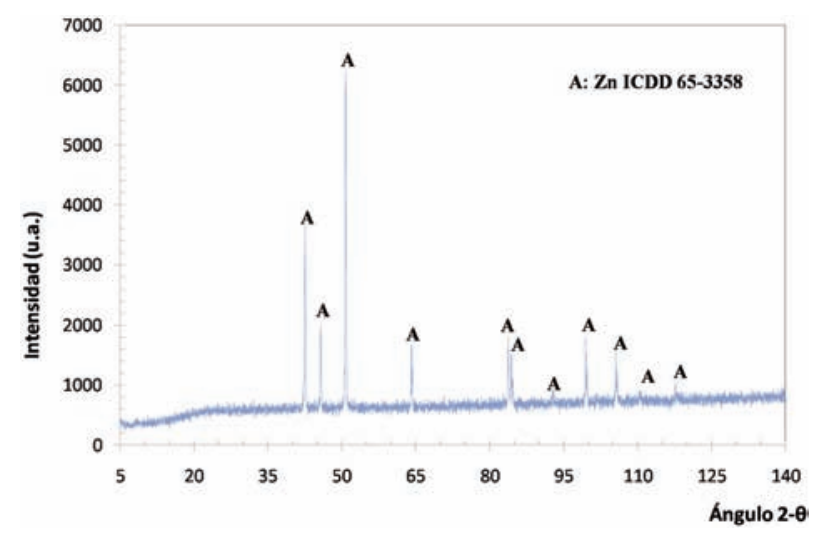

Figura 2. Difractograma de los polvos de galvanizado. Se indican los compuestos identificados mediante la comparación con las fichas JCPDS-ICDD correspondientes.

Figure 2. Diffractogram of the galvanizing dust. The compounds are identified through comparison with the corresponding JCPDS-ICDD records.

polvo al solidificar (Fig. 1 (a)). Bajo dichas condiciones termodinámicas, podría promoverse la oxidación ${ }^{[5]}$ parcial de la superficie de las gotas, la cual daría lugar a la formación minoritaria de óxido de zinc, que si bien no fue detectado mediante XRD, las medidas de densidad y el análisis químico por AS, inducen a pensar que este compuesto podría estar presente en los polvos de galvanizado. Cabe destacar, que los análisis químicos por AS indican que la totalidad de elementos cuantificados (Tabla II) se acerca al $99 \%$ en peso de la muestra, por lo que es posible inferir que el restante porcentaje es atribuible a la presencia de oxígeno, elemento que se encontraría asociado a los metales presentes en la muestra, especialmente al zinc.

Los análisis mediante FTIR, no arrojaron resultado alguno para los polvos de galvanizado, dado su carácter metálico. Esto permite inferir, en conjunto con los análisis químicos por AS y el análisis de fases por $\mathrm{XRD}$, que el zinc metálico se encuentra en este residuo como fase predominante, ya que los elementos metálicos son invisibles al infrarrojo ${ }^{[6]}$.

\subsection{Escoria "A"}

La escoria denominada como " $A$ " se genera en un proceso de galvanizado de piezas de acero grandes y geometría sencilla, llevado a cabo en una cuba de acero al carbono, en el que se mantiene al zinc fundido alrededor de $450^{\circ} \mathrm{C}$. Esta escoria es un material de aspecto heterogéneo, en forma de agregados pul- verulentos, de color predominantemente gris, y con una elevada presencia de partículas metálicas irregulares (gotas solidificadas de zinc). La densidad de este residuo fue calculada mediante picnometría y los resultados se muestran la tabla I.

Para realizar el análisis químico elemental de la escoria "A", se implementó la técnica de AS, cuyos resultados se muestran en la tabla II, resaltando una presencia mayoritaria de zinc en esta escoria. El resto de los elementos cuantificados no suma el $1 \%$ en peso, por lo que se le puede atribuir el porcentaje restante (aproximadamente $18 \%$ en peso) a la presencia de oxígeno, el cual se encuentra formando óxidos, primordialmente de zinc, como se revelarán más adelante los resultados de XRD.

La escoria "A" fue también estudiada mediante SEM, revelándose que la misma presenta una morfología tipo roseta, con distribución al azar, tal como se puede apreciar en la figura 3. Este tipo de morfología es característica del crecimiento cristalino en direcciones preferenciales y ha sido reportada en otros compuestos de zinc ${ }^{[7]}$. El rectángulo de la figura 3 indica el área en donde se realizó el microanálisis químico elemental semicuantitativo por EDS, cuyo espectro se muestra insertado dentro de la misma figura. La cuantificación del espectro se muestra en la tabla IV para esta escoria "A". Se destaca en el espectro la presencia de oxígeno, no obstante, este elemento no se cuantificó en los resultados finales reportados en la tabla IV. La técnica de EDS corrobora los análisis químicos por AS de este residuo, los cuales indican la predominancia del zinc en la muestra de estudio, pero también señalando la presencia de otros elementos que no fueron cuantificados mediante AS, como lo son el aluminio y el cloro, el primero de los cuales se origina de los elementos

\section{Tabla IV. Microanálisis químico elemental semicuantitativo por EDS (\% en peso) realizado sobre el área indicada en la figura 3 para la escoria "A"}

Table IV. Semiquantitative elemental chemical microanalysis by EDS (wt \%) performed on the area shown in figure 3 for the zinc ash "A"

\begin{tabular}{cc}
\hline Elemento & \% en peso \\
\hline $\mathrm{Zn}$ & 96,43 \\
$\mathrm{Al}$ & 0,91 \\
$\mathrm{Cl}$ & 2,65 \\
\hline
\end{tabular}




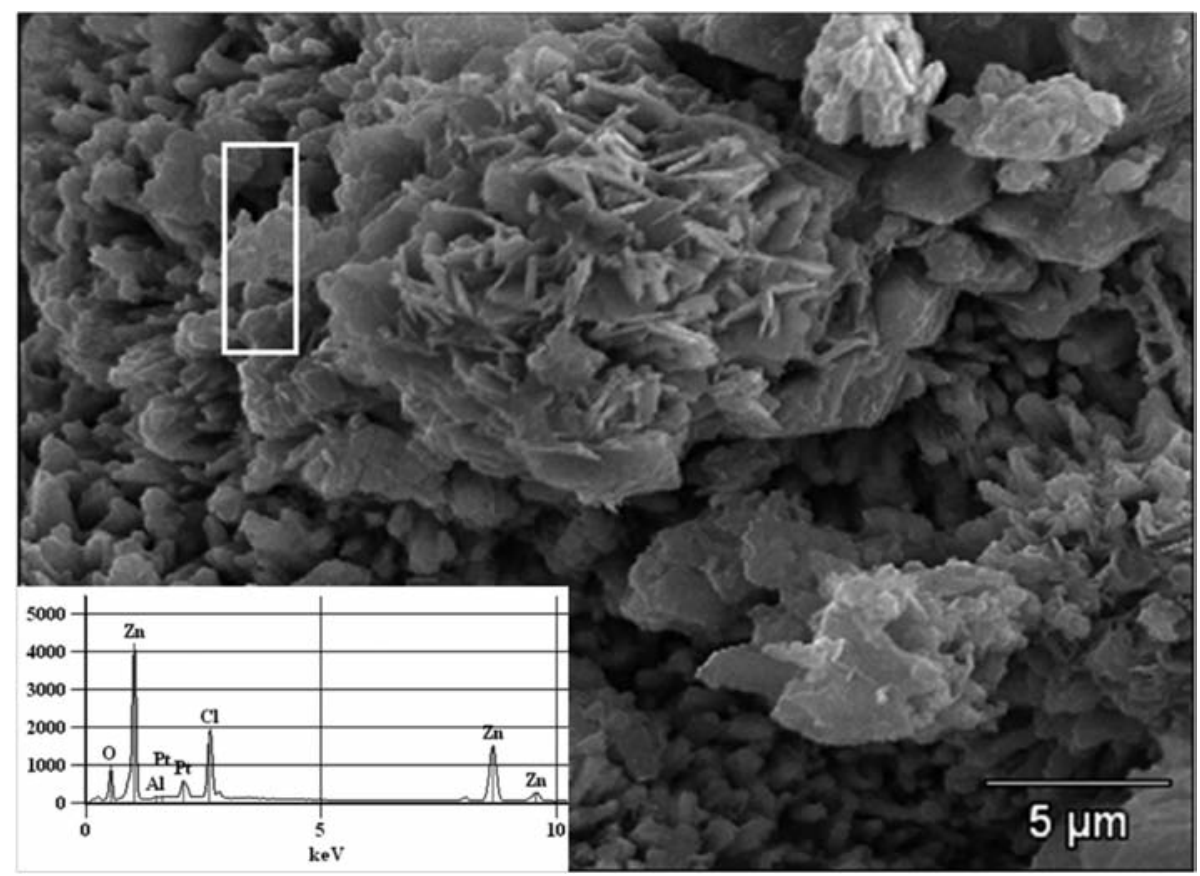

Figura 3. Imagen SEM de la escoria "A". Magnificación original de la imagen 4000X. El rectángulo indica el área donde se realizó el análisis químico elemental semicuantitativo por EDS, cuyo espectro se inserta en la figura.

\section{Figure 3. SEM image of zinc ash "A". Magnification of original image equal to 4000X. The square show the area where the semiquantitative EDS elemental chemical microanalysis was performed. The EDS spectrum is embedded.}

aleantes utilizados en el baño de zinc fundido y el segundo, proviene de las sales fundentes y activadoras de superficie, empleadas en el proceso industrial de galvanizado.

La identificación cualitativa de las fases cristalinas presentes en la escoria "A" se realizó mediante XRD. Los resultados que se muestran en el difractograma de la figura 4 indican la presencia de las siguientes fases: zinc metálico (Zn) y óxido de zinc $(\mathrm{ZnO})$. Otras fases posiblemente presentes en la escoria "A", que contengan los elementos restantes que aparecen en la tabla II y en la tabla IV, no fueron detectadas mediante esta técnica, probablemente debido a su menor presencia en comparación con las fases encontradas. Los resultados obtenidos son coincidentes con el análisis químico de la escoria "A". Por su parte, el espectro FTIR de este residuo se muestra en la figura 5 , en el que se nota la presencia de cuatro bandas características, las dos primeras correspondientes a los movimientos de estiramiento de los grupos $\mathrm{O}-\mathrm{H}$ presentes en la escoria, ubicadas en el rango de frecuencias entre 3600

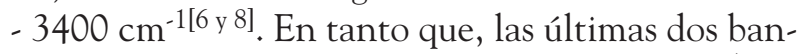
das, que se encuentran en 1507 y $1381 \mathrm{~cm}^{-1}$, se corresponden con los movimientos de flexión de dichos grupos $\mathrm{O}-\mathrm{H}^{[6}$ y 9$]$. Por otro lado, las bandas ubicadas a las frecuencias de 895 y $716 \mathrm{~cm}^{-1}$, pueden atribuirse las vibraciones de los grupos $\mathrm{O}-\mathrm{H}$ libres ${ }^{[6]}$. La presencia de estos grupos funcionales $\mathrm{OH}$ en la escoria puede ser explicada por dos motivos, el primero es la eventual presencia de agua físicamente adsorbida en la superficie de la escoria y el segundo es la presencia de grupos $\mathrm{OH}$ de sustitución presentes en la estructura del óxido de zinc $(\mathrm{ZnO})$. Finalmente, la banda de menor frecuencia, ubicada a $478 \mathrm{~cm}^{-1}$, es propia de los enlaces M-O de los óxidos minerales, en este caso, dicha contribución al espectro infrarrojo obedece a los enlaces $\mathrm{Zn}-\mathrm{O}$ en el óxido de zinc $(\mathrm{ZnO})^{[9-11]}$.

\subsection{Escoria "B"}

Este residuo sólido de la cuba de galvanizado, denominado escoria "B", es un material heterogéneo de color amarillento, con tendencia a formar aglomerados fácilmente separables por medios físicos. La 


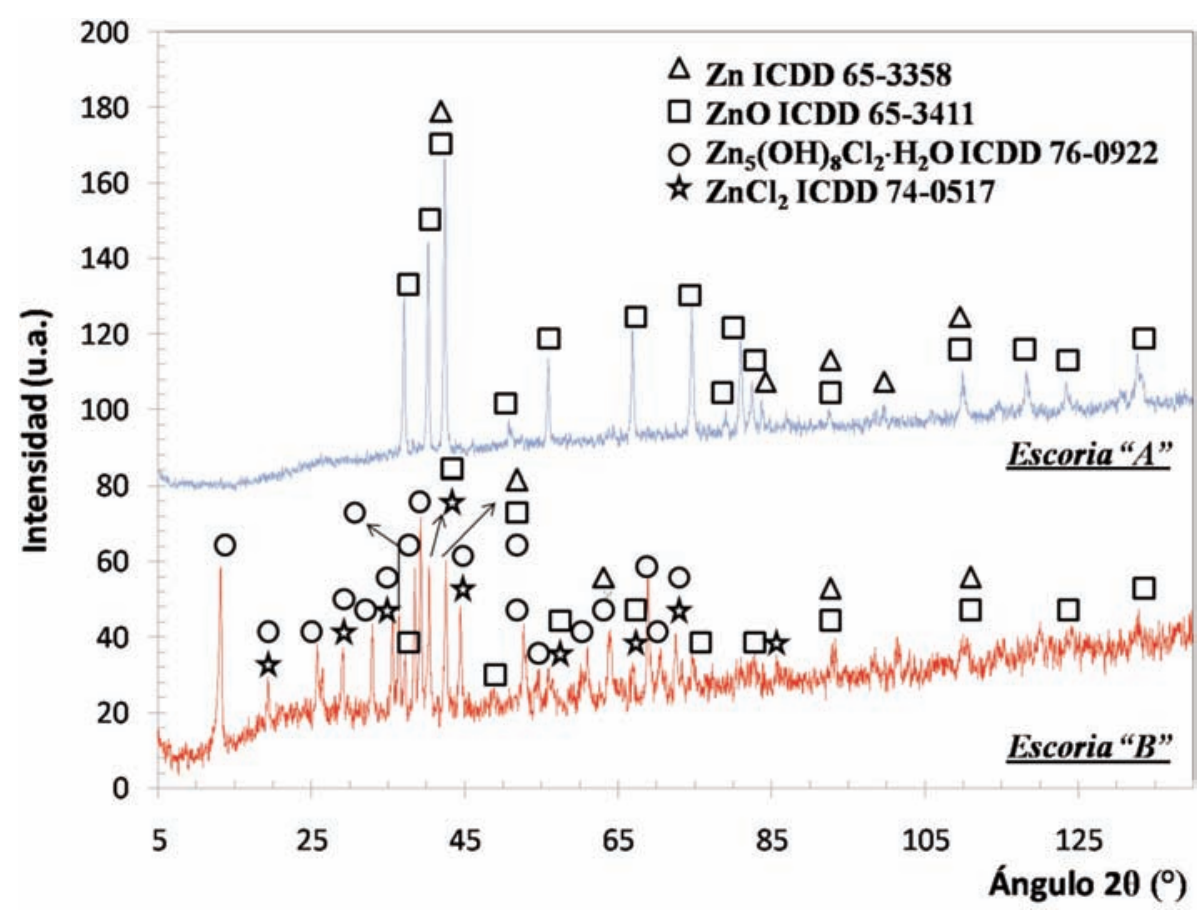

Figura 4. Difractogramas de las escorias "A" y "B". Se indican los compuestos identificados mediante la comparación con las fichas JCPDSICDD correspondientes.

Figure 4. Diffractograms of the zinc ashes " $A$ " and "B". The compounds were identified through comparison with the corresponding JCPDSICDD records.

densidad de esta escoria se calculó por picnometría y los resultados se muestran la tabla I, en donde se evidencia una clara diferencia con la densidad de la escoria "A", lo que puede tomarse como un primer indicativo de la diferencia de sus composiciones en

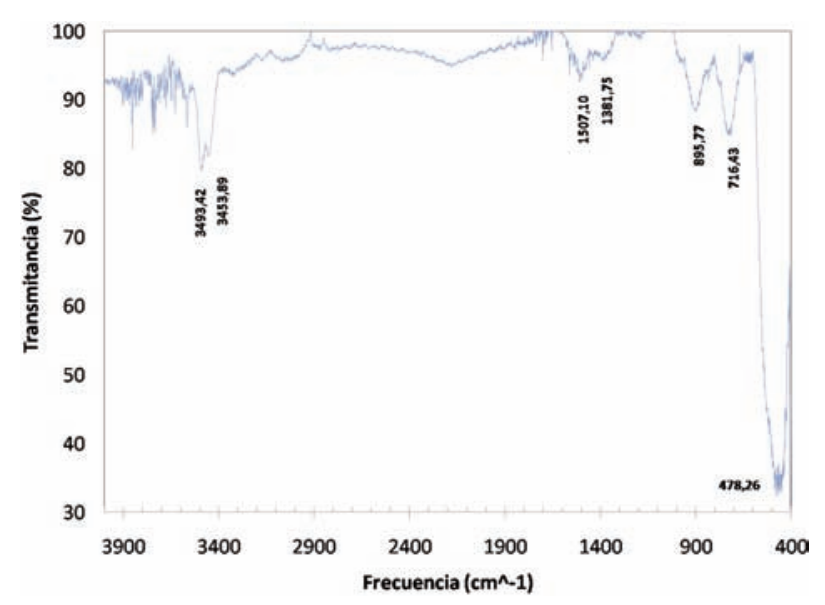

Figura 5. Espectro FTIR de la escoria "A".

Figure 5. FTIR spectrum of the zinc ash "A". cuanto a fases se refiere y en las proporciones relativas de éstas.

La química elemental de esta escoria se obtuvo por AS y los resultados pueden observarse en la tabla II. Al igual que en la escoria "A", el zinc es el elemento presente en mayor proporción en la escoria "B" (75\% en peso). Sin embargo, el resto de los elementos cuantificados se encuentra en cantidades superiores en comparación con estos mismos presentes en la escoria "A", en especial, el hierro y el manganeso, los cuales son hallados en la escoria "B" en proporciones relativamente elevadas. Estos dos últimos elementos provienen estrictamente de las piezas de acero a galvanizar, ya que, en la planta que genera esta escoria, la cuba que contiene al zinc líquido se encuentra cubierta por ladrillos refractarios que en la práctica reaccionan muy poco con baño de zinc. Las cubas cerámicas permiten trabajar a temperaturas de zinc líquido más elevadas, que pueden rondar los $560^{\circ} \mathrm{C}$, lo cual facilita el galvanizado de volúmenes importantes de piezas pequeñas de geometría compleja a granel, esto significa que el zinc en este proceso está, en promedio, unos $120^{\circ} \mathrm{C}$ más caliente que en su contraparte que es la cuba de acero al 


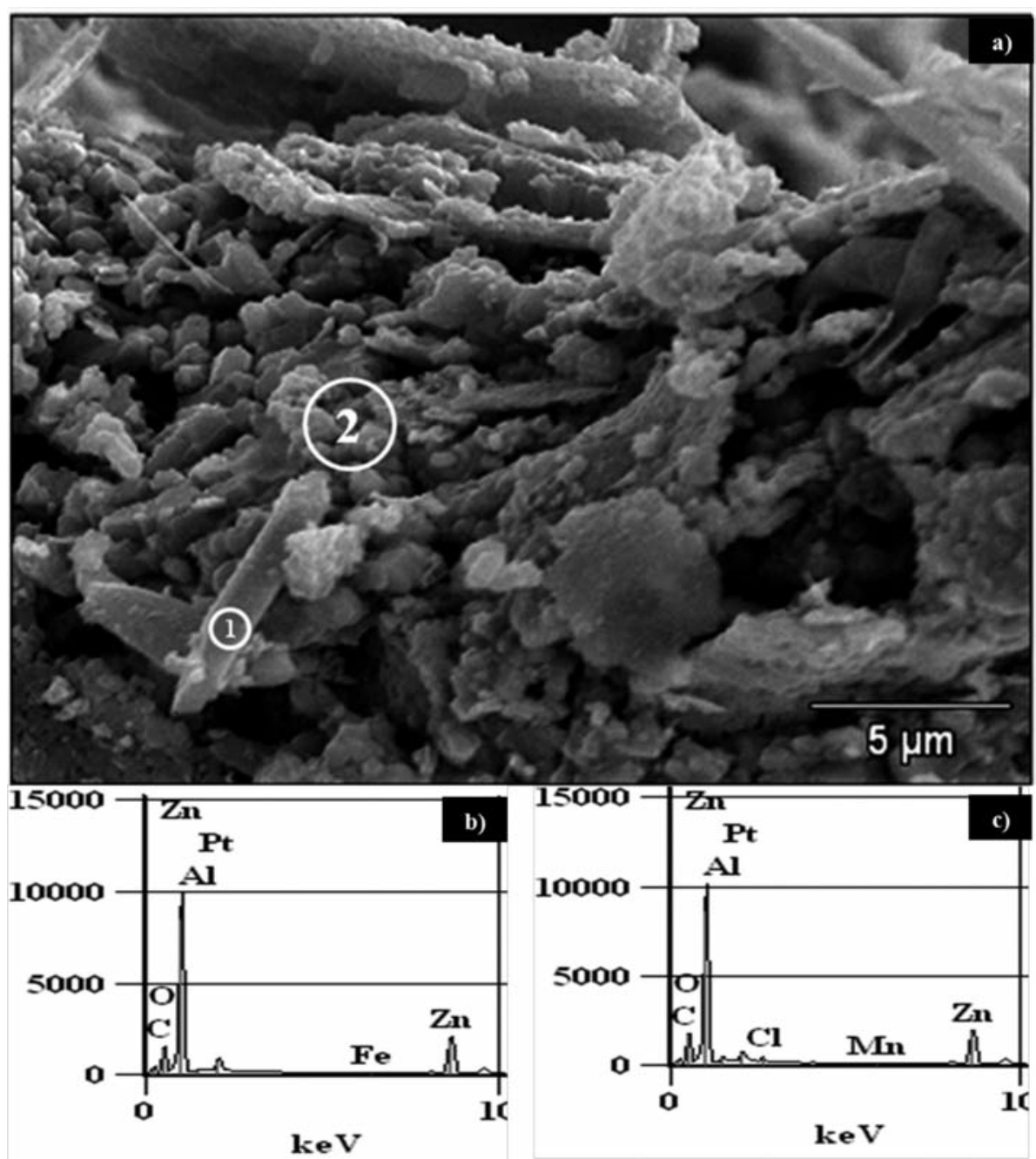

Figura 6. (a) Imagen SEM de la escoria "B". Magnificación original 5000X. Las figuras 6 (b) y 6 (c) indican, respectivamente, los espectros EDS resultantes de los análisis realizados sobre las circunferencias 1 y 2 en la figura.

Figure 6. (a) SEM image of zinc ash " $B$ ". Magnification of original image equal to 5000X. figures 6 (b) and 6 (c) show, respectively, the EDS spectra of the analysis performed on circles 1 and 2 in figure.

carbono. Bajo estas condiciones, el zinc líquido es más reactivo y ataca en mayor medida a la superficie del acero, favoreciendo que se incorporen al baño cantidades importantes de hierro y manganeso, los cuales se ven reflejados también en la escoria que se genera en esta clase de proceso productivo (escoria "B").

Los resultados del análisis morfológico de la escoria "B" se encuentran resumidos en la figura 6 , cuya imagen refleja una morfología de tipo hojuelas irregulares, la cual está constituida por cristales elongados. Este tipo de morfología es similar a la encontrada en algunos compuestos de zinc reportados en la literatura ${ }^{[12]}$. Las circunferencias que aparecen en la figura 6, representan las zonas en donde se realizó el análisis químico elemental semicuantitativo por EDS, el cual arrojó los resultados que se pueden apreciar en la tabla $\mathrm{V}$ normalizados al $100 \%$ en peso. Al igual que con la escoria "A", se destaca la detección de oxígeno mediante EDS, tal como reflejan los espectros de las figuras 6 (b) y (c), a pesar de no figurar entre los elementos presentes en la tabla $\mathrm{V}$ ya que el mismo no se consideró en la cuantificación definitiva. La técnica de EDS permitió detectar la presencia de otros elementos químicos que no fueron determinados mediante AS, tales como el cloro y el aluminio. Al igual que en el caso de los polvos 
Tabla V. Microanálisis químico elemental semicuantitativo por EDS (\% en peso) realizado sobre las zonas indicadas en la figura 5 para la escoria "B"

Table V. Semiquantitative elemental chemical microanalysis by EDS (wt \%) performed on the areas shown in figure 5 for zinc ash "B"

\begin{tabular}{ccc}
\hline & Zona 1 & Zona 2 \\
\hline $\mathrm{Zn}$ & 98,77 & 95,89 \\
$\mathrm{Al}$ & 0,82 & 2,52 \\
$\mathrm{Fe}$ & 0,41 & - \\
$\mathrm{Mn}$ & - & 0,15 \\
$\mathrm{Cl}$ & - & 1,44 \\
\hline
\end{tabular}

y de la escoria "A", la presencia de estos elementos se explica por las condiciones inherentes del proceso de galvanizado en caliente (adición de aluminio al baño y uso de sales modificadoras de la superficie).

Las fases cristalinas presentes en la escoria "B" fueron identificadas cualitativamente mediante XRD. Los resultados de la implementación de la técnica se muestran en el difractograma de la figura 4 , en donde se destaca la presencia de máximos de difracción característicos de las siguientes fases: zinc metálico $(\mathrm{Zn})$, óxido de zinc $(\mathrm{ZnO})$, hidroxicloruro de zinc $\left(\mathrm{Zn}_{5}(\mathrm{OH})_{8} \mathrm{Cl}_{2} \times \mathrm{H}_{2} \mathrm{O}\right)$ y cloruro de zinc $\left(\mathrm{ZnCl}_{2}\right)$. Los dos primeros compuestos aparecen también en la composición de la escoria "A", mientras que los dos últimos son detectados únicamente en la escoria "B" y su origen puede atribuirse a los compuestos residuales provenientes de los procesos previos de limpieza de las piezas, los cuales reaccionan con el zinc del baño fundido para originar compuestos químicos con estos elementos.

Como último análisis se realizó un estudio de FTIR en una muestra de la escoria "B". Los resultados se pueden apreciar en el espectro infrarrojo de la figura 7, donde se observa que, al igual que con la escoria "A", se distinguen bandas características entre $3600-3400 \mathrm{~cm}^{-1}$, correspondientes a los movimientos de estiramiento de los grupos $\mathrm{O}$-H físicamente adsorbidos $^{[6}$ y $^{8]}$. Por otro lado, las bandas de frecuencia comprendidas entre $1605-1042 \mathrm{~cm}^{-1}$, pueden atribuirse a las vibraciones de flexión de los enlaces de los grupos $\mathrm{O}-\mathrm{H}$ presentes en la muestra ${ }^{[6}$ y 9$]$, así como también pueden atribuirse otras vibraciones de los mismos grupos a las bandas ubicadas en las frecuencias de 908 y $720 \mathrm{~cm}^{-16]}$. Adicionalmente, se pueden atribuir las bandas de frecuencia de 626 y $525 \mathrm{~cm}^{-1}$, a vibraciones relacionadas con el cloro presente en el hidroxicloruro de zinc ${ }^{[6]}$, identificado por XRD. Finalmente, cabe acotar que al igual que en la escoria

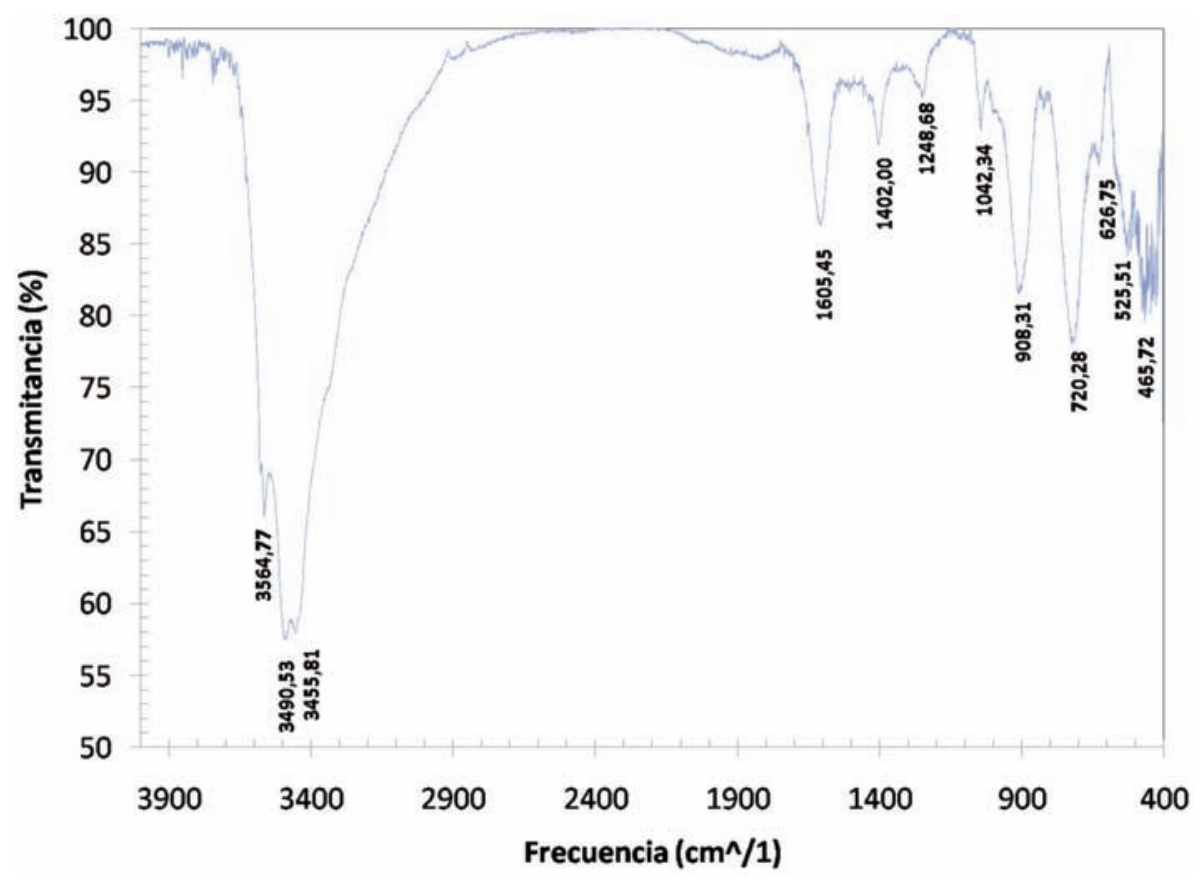

Figura 7. Espectro FTIR de la escoria "B".

Figure 7. FTIR spectrum of the zinc ash "B". 
"A", la escoria "B" presentó una banda de frecuencia

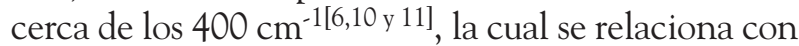
las vibraciones características de los enlaces $\mathrm{M}-\mathrm{O}$, en cuyo caso particular, el metal presente en el caso de estudio es el zinc.

\section{IMPLICACIONES DE ESTOS RESULTADOS DE CARA AL PROCESO DE VALORIZACIÓN DE LOS RESIDUOS SÓLIDOS DE LA INDUSTRIA DEL GALVANIZADO}

A pesar de la presencia mayoritaria de zinc metálico en los polvos residuales de galvanizado, este material no puede reciclarse directamente en forma de aporte al baño de zinc fundido, debido a su consistencia y tamaño micrométrico, ya que el mismo resultaría muy fácilmente oxidable y difícil de sumergir en el zinc líquido, a menos que se someta a un proceso previo de compactación. Por otro lado, los polvos de galvanizado podrían ser utilizados como materia prima en industrias que requieran de zinc metálico de tamaño micrométrico, como puede ser el caso de la industria extractiva de oro, la cual requiere polvo de zinc durante el proceso de cementación ${ }^{[13]}$.

En lo que respecta a las escorias estudiadas, ambas poseen una fracción metálica, en principio, directamente aprovechable luego de su separación física, bien dentro del propio proceso de galvanizado o como chatarra rica en zinc en algún proceso de fundición. En la actualidad, este aprovechamiento del zinc metálico presente en la escoria es realizado por las empresas que suministran los lingotes de zinc a las galvanizadoras. A pesar de esto, las escorias cuentan con una importante fracción de carácter no metálico, la cual es descartada, ya que no resulta ser fácilmente aprovechable. Sin embargo, dado el elevado contenido de compuestos de zinc que tiene esta fracción, resulta conveniente explorar vías de valorización que sirvan para convertir dichas fracciones en materias primas aprovechables en otros procesos industriales. Debido a que las mismas contienen $\mathrm{ZnO}$, pueden plantearse procesos de separación o purificación, que permitan recuperar este compuesto, el cual es ampliamente utilizado en otras industrias tales como la de los elastómeros, los cosméticos, fármacos y alimentos, entre otras $^{[14]}$.

Adicionalmente, otros productos de zinc con aplicaciones tecnológicas podrían sintetizarse de forma sencilla a partir de estos residuos ricos en zinc, tal es el caso del sulfato de zinc $\left(\mathrm{ZnSO}_{4}\right)$ y el complejo ZnEDTA, empleados como componentes de los fertilizantes para suplir los bajos niveles de zinc en suelos en los que se producen cultivos tales como el arroz ${ }^{[15]}$.
En el mismo orden de ideas, también se emplean sales orgánicas de zinc para incrementar la biodisponibilidad de este mineral en los organismos vivos, mediante suplementos nutricionales basados en gluconato o citrato de zinc ${ }^{[16]}$. Esto abre las posibilidades de un eventual tratamiento hidrometalúrgico de las escorias de galvanizado con ácidos orgánicos y otros agentes complejantes, con el fin de precipitar sales con mayor valor agregado, utilizables por las industrias antes mencionadas.

\section{CONCLUSIONES}

- En el presente trabajo se caracterizaron fisicoquímicamente polvos y escorias procedentes de la industria de galvanizado en caliente por inmersión. Se encontró que los polvos estuvieron constituidos principalmente por zinc metálico (98\% en peso) con morfología esferoidal y un tamaño medio de partícula de $16,5 \mu \mathrm{m}$, los cuales podrían ser aprovechables en procesos tales como la cementación de oro. Por su parte, ambas escorias de galvanizado presentaron una cantidad de zinc similar, (71 \% escoria “A” y 75 \% escoria "B", en peso). No obstante, la cantidad de hierro en la escoria "B" ( $8,3 \%$ en peso) supera ampliamente a la de la escoria "A" (0,3\% en peso), lo cual se atribuye a la variación de las temperaturas empleadas en los procesos de galvanizado que generan cada una de estas escorias.

- El análisis por XRD permitió identificar Zn (metálico) y $\mathrm{ZnO}$ en la escoria "A", mientras que, en la escoria "B", se detectó la presencia de $\mathrm{Zn}$, $\mathrm{ZnO}, \mathrm{Zn}_{5}(\mathrm{OH})_{8} \mathrm{Cl}_{2} \times \mathrm{H}_{2} \mathrm{O}$ y $\mathrm{ZnCl}_{2}$.

- Los estudios mediante FTIR indicaron que ambas escorias presentaron la incorporación de agua en su estructura.

- Finalmente, tomando como base los resultados obtenidos, se argumentó la aplicabilidad de estas escorias en diversas industrias, tales como la agrícola, farmacéutica y de alimentos, siempre y cuando las mismas se sometan a un proceso de valorización previo.

\section{REFERENCIAS}

[1] N. Durán, Tesis de Licenciatura en Ingeniería de Materiales, Departamento de Ciencias de los Materiales, Universidad Simón Bolívar, 2010.

[2] X. Castells, Reciclaje de Residuos Industriales, Ed. Díaz de Santos S. A., Madrid, España, 2000, pp. 1-2, 15-18, 27-29, 45-48. 
[3] C. Escudero, Tesis Doctoral, Departament d' Enginyeria Química, Agrària i Tecnologia Agroalimentaria, Universitat de Girona, 2009.

[4] L. Korb y D. Olson, Corrosion, ASM Handbook, Volume 13, ASM International, 4ta. Edición, EE.UU., 1992, pp. 959-974.

[5] N. Birks, G.Meier y F. Petit, Introduction to HighTemperature Oxidation of Metals, Cambridge University Press, 2da. Edición, Inglaterra, 2006, pp. 23.

[6] B. H. Stuart, Infrared Spectroscopy: Fundamentals and Applications, John Wiley \& Sons Ltd., Chichester, Inglaterra, 2004, pp. 26-27, 96-99.

[7] M. Saheer, M. Alam, M. Seok Jon y O. Yang, Electrochim. Acta 53 (2008) 7869-7874.

[8] M. Yeganeh, J. Alloy Compd. 491 (2010) 420422.

[9] B. M. Keyes, L. M. Gedvilas, X. Li y T. J. Coutts, J. Cryst. Growth 281 (2005) 297-302.
[10] C. Pholnak, C. Sirisathitkul y D. J. Harding, J. Phys. Chem. Solids. 72 (2011) 817-823.

[11] O. Kammori, N. Yamaguchi y K. Sato, Jpn. Analyst. 16 -10 (1967)1.050-1.055.

[12] G. Zhang, S. Yu, Y. Yang, W. Jiang, S. Zhang y B. Huang, J. Cryst. Growth 312 (2010) 1.8661.871 .

[13] P. Navarro, C. Vargas, R. Álvarez y F. J. Alguacil, Rev. Metal. Madrid 41 (2005) 12-20.

[14] J. O. Nriagu, Zinc in the environment. Part I: Ecological Cycling, John Wiley \& Sons Ltd., Burlington, Canadá, 1980, pp. 4, 33-35, 39-45, 52-57, 337-343, 347-349.

[15] I. Cakmak, J. Trace. Elem. Med. Bio, 23 (2009) 281-289.

[16] M. Salgueiro, M. Zubillaga, A. Lysionek, M. Sarabia, R. Caro, T. De Paoli, A. Hager, E. Ettlin, R. Weill y J. Boccio, Nutrition 16-9 (2000) 762-766. 